For Submission to the:

Journal of Power Sources

Corresponding Author: J. Peter Hensel Phone: (304) 285-4073 FAX: (304) 285-4469

\title{
EFFECTS OF CELL-TO-CELL FUEL MAL-DISTRIBUTION ON FUEL CELL PERFORMANCE AND A MEANS TO REDUCE MAL-DISTRIBUTION USING MEMS MICRO-VALVES
}

\author{
J. Peter Hensel ${ }^{1}$, Randall S. Gemmen ${ }^{1}$, Jimmy D. Thornton ${ }^{1}$ \\ Jeffrey S. Vipperman², William W. Clark², Brian A. Bucci ${ }^{2}$ \\ ${ }^{1}$ National Energy Technology Center, P. O .Box 880, Morgantown, WV, USA; E-mail: j.hensel@netl.doe.gov \\ ${ }^{2}$ Dept. of Mechanical Engineering, University of Pittsburgh, Pittsburgh, PA; E-mail: jsv@pitt.edu
}

Abstract

\begin{abstract}
Achieving uniform flow among the cells of a fuel cell stack plays a significant role in being able to operate at maximum capability and efficiency. This paper presents experimental data showing the importance of cell-to-cell fuel flow balancing on fuel cell performance, and a Fuel Cell Energy Management (FCEM) technique that has demonstrated the ability to improve stack performance. In a specially instrumented four-cell polymer electrolyte fuel cell that allows external control of the air, fuel, and water cooling flows to each cell, fuel to a single cell was reduced. V-I curves collected under these unbalanced conditions are compared to curves collected when the fuel flow to each cell was balanced. Reducing the fuel flow to a single cell by $11 \%$ decreased the V-I curve cutoff load by $10 \%$ - demonstrating the degree of negative effect that unbalanced fuel flows can have on stack performance. Typical fuel cell stacks have no dynamic means to keep flows in the stack balanced between the cells, but through the use of custom-built, piezoelectric micro-valves, a simple flow control strategy, and this custom 4-cell laboratory stack, the performance benefits of FCEM fuel flow balancing with respect to overall V-I performance was demonstrated. With the external FCEM technique, the stack's maximum fuel utilization was increased by as much as 5 percent.
\end{abstract}

Keywords: PEMFC; MEMS; micro-valve; fuel cell energy management; flow imbalance

\section{Introduction}

Achieving uniform reactant distribution, both cell-tocell and within individual cells of a fuel cell stack, provides a serious challenge to successful fuel cell design and operation. Studies have shown that flow distribution variances from the internal reactant manifolds to the individual cells of the stack, especially at the ends of the manifolds, are not uncommon, and can lead to a shortage of mass flow reaching these cells (McGarry et al. [1]). This is true regardless of the type of fuel cell (polymer electrolyte, solid oxide, etc.) Fuel cell engineers attempt to achieve uniform reactant distribution by designing cells/stacks with tight manufacturing tolerances and sufficient pressure drop so that flow can be maintained uniform throughout the stack over a wide range of operating conditions. Determining the correct dimensions for the internal flow passages (manifolds and anode/cathode channels) can be a costly engineering exercise in both analysis and testing. Even with the correct dimensions known, achieving a low cost mass produced fuel cell system having tight tolerances may be difficult to achieve. Furthermore, in polymer electrolyte, or proton exchange membrane fuel cells (PEMFCs) problems can arise when the electrochemically generated water vapor condenses onto the channel surfaces (cathode flooding) thereby restricting or even blocking the flow through the channel and reducing the amount of oxygen transported to the cathode reaction zone. Similar problems can also arise on the anode, where anode water vapor condenses on the diffusion layer and inhibits hydrogen diffusion to the anode reaction zone. Water condensation problems can arise at different operating points for a stack, depending on the temperature, pressure, anode and cathode flow rates, and power drawn from the stack, and can be especially problematic if the system is to be used under a wide range of operating conditions. When such problems occur, the associated cell performs poorly (generates low or fluctuating voltage and current) and begins to overheat. Operating under these conditions for too long will damage the membrane. In addition, these situations can go unnoticed by control strategies that only monitor the overall stack voltage, especially in high voltage stacks that incorporate many cells.

A number of techniques to improve cell/stack performance have been proposed and studied. For instance, for PEMFCs, Lee et al. [2] showed the effectiveness of a thermal and water management technique to reduce cell-to-cell voltage variations in a stack. The technique requires excess fuel flow, over 1.3 times the required fuel stoichiometry, to provide adequate flow to each cell in the stack with the unused fuel being re-circulated back to the inlet to keep the fuel utilization high. In a similar technique, called the anode water removal technique, Voss et al., [3], uses an increased pressure drop across the cell and an increased amount of re-circulated fuel. Some research is attempting to remedy the water condensation problems by using higher temperature components so that water condensation, and therefore water blockage in the cells, no longer occurs (Mallant [4]). 
In spite of these various approaches to improving PEMFC stack performance, engineers are forced to derate the maximum power output and efficiency due to the remaining uncertainty caused by stack manufacturing tolerances as well as the ever present random process of water condensation over the surface of a cell. This occurs, for example, when the total flow (fuel or air) is increased by an amount needed to force enough flow to a flow-limited cell. Doing so, however, results in low fuel efficiency since more excess fuel is emitted from the stack. It is therefore apparent that the uncertainty in the amount of flow to a single cell in a stack (due to both assembly tolerances and the random processes within the stack) directly limits the performance of a stack. Therefore, it would be worth considering other methods that can be used after stack assembly (and even during operation) to correct for flow mal-distribution, whether they be caused by the stack manufacturing process or from normal operating conditions.

An alternative approach to managing and ensuring adequate fuel flow to each cell in a PEMFC is under investigation at the National Energy Technology Laboratory (NETL) supported by the Advanced Research and Technology Development Program. This approach is called the Fuel Cell Energy Management (FCEM) technique. The objective of the FCEM research is to increase fuel cell performance by dynamically balancing the cell-to-cell flow distribution in the stack thereby allowing the stack to be safely and reliably operated at as high a fuel utilization as possible. However, using conventional valves to perform flow distribution, while acceptable for experiments in the lab, can be very expensive if not entirely impractical in the commercial world. Instead, Micro-Electro-Mechanical Systems (MEMS) technology can provide micro-valves for dynamic, individual flow control at a fraction of the cost of conventional technology, and the benefits of this technique could apply to any fuel cell type, although this work has been focused on PEMFCs which are more easily instrumented for study. Integration of micro-valves along the internal fuel feed manifold of the stack to control the fuel flow to each cell is believed possible, but until such internal control can be achieved, an external version of the FCEM concept has been investigated and the performance results, in comparison to a common manifold (CM) stack, are presented.

The focus of this paper is to experimentally demonstrate the negative effect of cell-to-cell fuel maldistribution, and the positive performance benefits that fuel-based FCEM offers over conventional common manifold stacks. The paper begins by presenting the experimental approach taken for this project. It presents: (1) the methodology used in conducting common manifold stack experiments to provide a performance baseline with the FCEM work as well as the results of these experiments; (2) the methodology used in conducting fuel flow experiments to show the effect of fuel flow mal-distribution on stack performance along with the associated results; (3) the development of a micro-valve suitable for PEMFC environments; and finally, (4) an implementation of the FCEM technique developed using the aforementioned micro-valves - the Micro-Valve Flow Control System (MVFCS). This is followed by a discussion of the results, a list of conclusions, and a mention of upcoming future work.

\section{Experimental approach and results}

For this work, a PEM test facility suitable for performing individual cell flow control studies with a 4cell stack was constructed. In the first study, a standard common manifold stack was developed (which is representative of most fuel cell configurations under development today) to characterize the typical performance of a stack that has no on-line control system for adjusting individual cell flows. Then, a unique stack was built to facilitate control of fuel, air and cooling water to each cell, using specially designed flow separator plates, along with the same cathode and anode plates and MEA's as were used in the common manifold stack. This unique stack design is herein referred to as the Isolated Cell Stack (ICS). Two unique fuel delivery systems were studied using the ICS. The first, called the Mass Flow Control System (MFCS), used 4 individual mass flow controllers to control the fuel flow to each cell. With this system, the effects of cell-to-cell mal-distribution were evaluated. The second system, called the Micro-Valve Flow Control System (MVFCS), used 4 micro-valves (developed at the University of Pittsburgh) to manipulate the 4 individual fuel flows to the ICS. This second system was employed to evaluate FCEM techniques, or more specifically, dynamic flow control techniques that could be used in internal micro-valve systems. Each of these test systems is described in the following subsections.

\subsection{Common manifold stack experiments}

For this part of the experimental analysis, a 4-cell "common manifold" PEMFC stack, in which all four cells received fuel from a common manifold (see Figure 1) and hence depended on local flow resistance to determine the flow distribution, was designed and assembled. Such a flow configuration is representative of the "passive flow management" fuel cell systems currently in development and commercialization. This fuel cell system was tested on a laboratory test stand suitable for controlling and testing the critical system parameters of pressure, temperature, relative humidity, cooling, and fuel and air flows.

A schematic of the flow plate design (which, except for the depth of the flow channels, was the same for both the anode and the cathode) is shown in Figure 2. Each cell's active area is $0.01336 \mathrm{~m}^{2}(11.51 \mathrm{~cm} \times 11.62 \mathrm{~cm})$. The anode and cathode plates are made from synthetic graphite, each having 15 equally-spaced, serpentine flow 


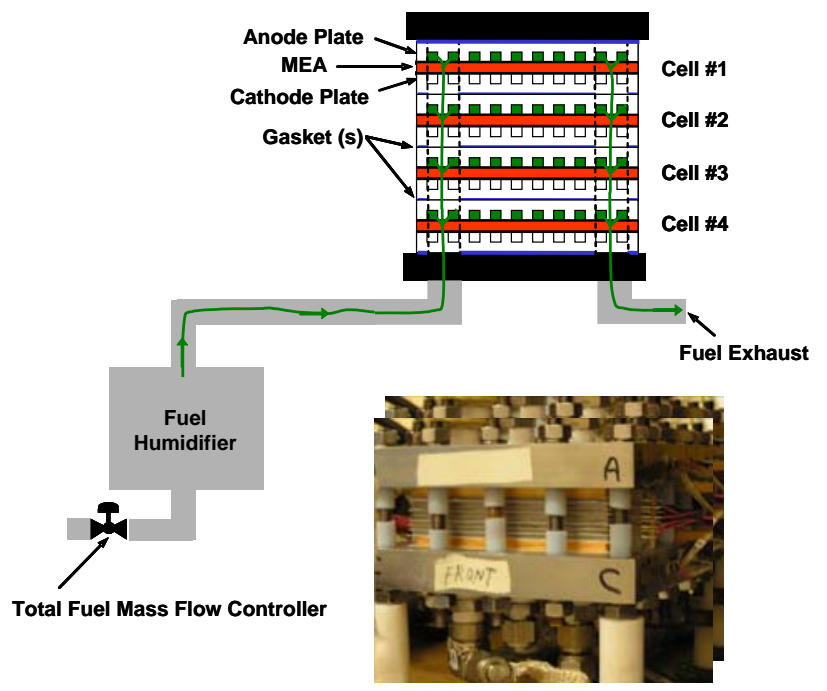

Fig. 1. Conventional common manifold stack fuel configuration and photo.

channels $1.59 \mathrm{~mm}$ wide. The air flow channels on the cathode plates are $1.59 \mathrm{~mm}$ deep. The fuel flow channels and the water-cooling channels are $0.795 \mathrm{~mm}$ deep and exist on opposite sides of the anode plates. The plate thickness is approximately $2.4 \mathrm{~mm}$. The Membrane Electrode Assemblies (MEAs) (with catalyst) have a membrane thickness of $0.051 \mathrm{~mm}$. The anode specifications are: ELAT/std/DS/Vulcan XC-72, 20\% Pt, $0.4 \mathrm{mg}$ per $\mathrm{cm}^{2} / 0.6-0.8$ Nafion Ionomer Loading; and the specifications for the cathode are: ELAT/std/SS/Vulcan XC-72, $40 \% \mathrm{Pt}, 0.4 \mathrm{mg}$ per $\mathrm{cm}^{2} /$ 0.6-0.8 Nafion Ionomer Loading. The four cells are bolted between two temperature-controlled, $2.86 \mathrm{~cm}$ thick, stainless steel end plates. Air and fuel flows to the stack are metered and controlled by mass flow controllers, routed through temperature-controlled humidifier vessels that maintain the flows at a $100 \%$ relative humidity, and channeled via temperature controlled feed lines into the air and fuel inlets of the stack. Water cooling, through the anode plates, is controlled by the speed of the water pump. Water cooling was included since the test plans involved running the stack up to the highest possible current density. A flow rate of approximately 0.2 liters/min was used to keep the cell temperatures from varying too far from the nominal 80 degrees $C$.

A series of preliminary tests were run on this system to determine essential flow humidification parameters, resolve stack heating/cooling issues, and fine-tune the instrumentation and control requirements. Stack performance was then characterized at 3 different fuel flow rates - 2.8, 3.2, and 3.6 SLPM of $\mathrm{H}_{2}$. Three tests at each of these fuel flow rates were conducted. The stack voltage vs. load (V-I) and the standard deviation of the stack voltage versus load for the tests where the minimum and the maximum fuel utilizations were observed are presented in Figures 3-5, along with data from the later micro-valve flow control system tests - just for comparison purposes. Figure 3 shows the data from the tests run with a total fuel flow rate of 2.8 SLPM. Figure 4 shows the data from the 3.2 SLPM tests and Figure 5 the 3.6 SLPM tests. The standard deviation values were calculated while at steady-state conditions over the 200 second time period immediately prior to recording the stack voltage. (This is true of any standard deviation data reported in this paper.) Figures 3-5 also include the percent fuel utilization values, on the secondary $\mathrm{x}$-axis, for the fuel delivery rates used.

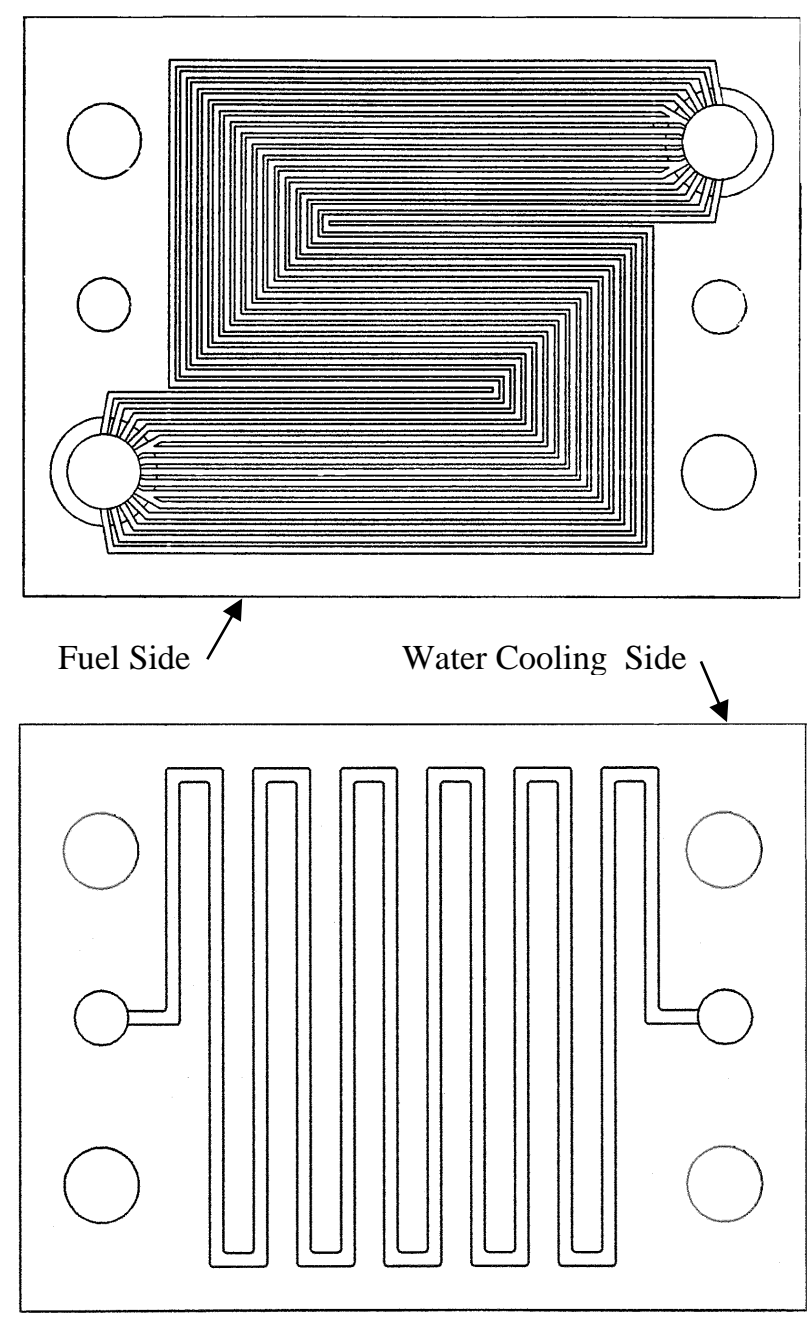

Fig. 2. Schematic of flow channels on anode plate.

\subsection{Cell-to-cell flow control study}

The FCEM technique was used to resolve the effects of cell-to-cell flow mal-distribution problems. To study this, a new 4-cell stack (the ICS) was built using the same anode, cathode and MEA hardware as in the common manifold tests. This stack incorporates thick, steel separator plates between the four cells allowing individual control of air, fuel, and water cooling flows to each cell. In this FCEM configuration, all cell inlet flows can be individually controlled from outside the stack. However, the outlets from each cell empty into internal stack 


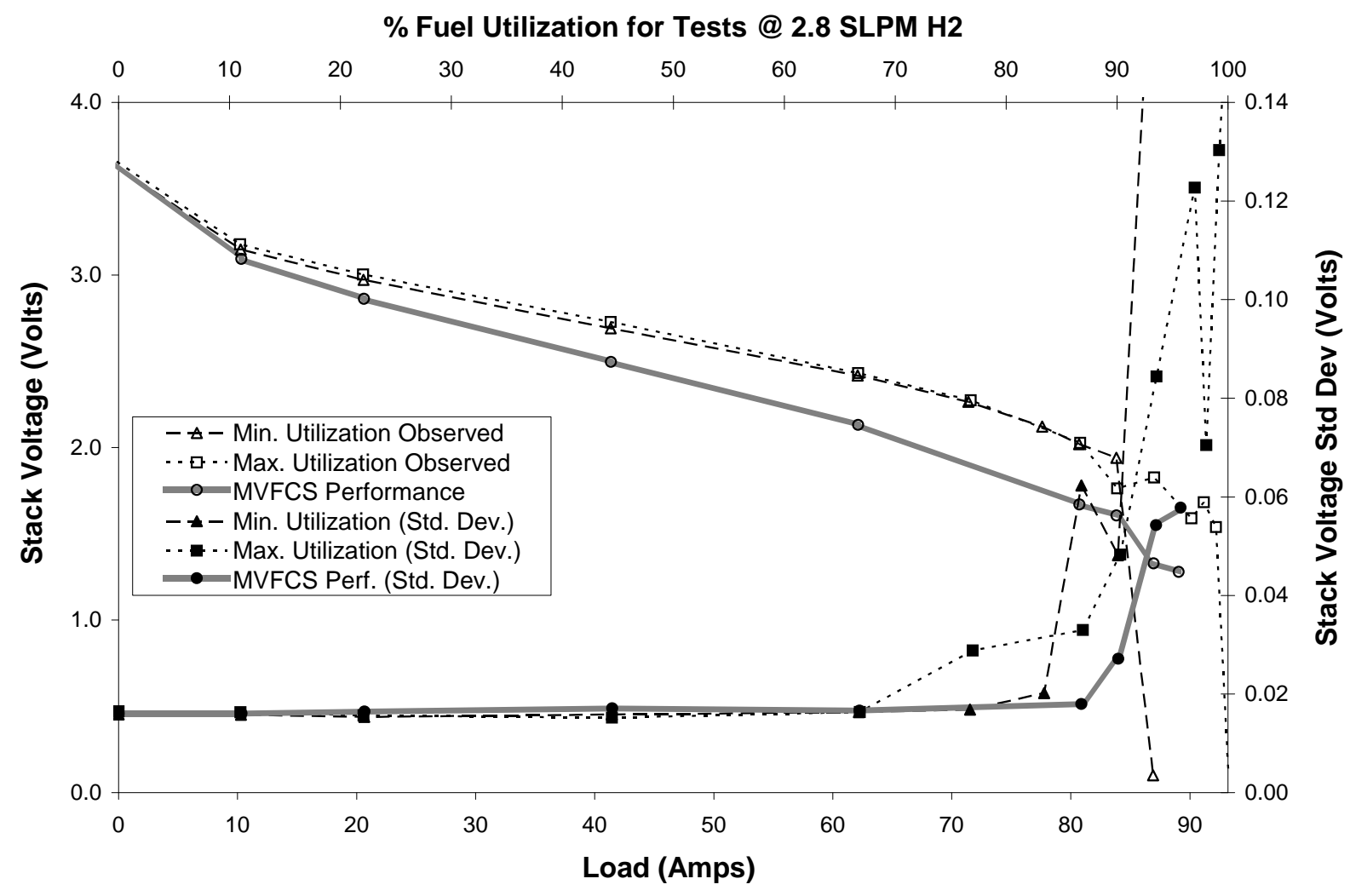

Fig. 3. Common manifold stack performance compared with MVFCS stack performance at a fuel flow rate of 2.8 SLPM - stack V-I curves and standard deviations of the stack voltages.

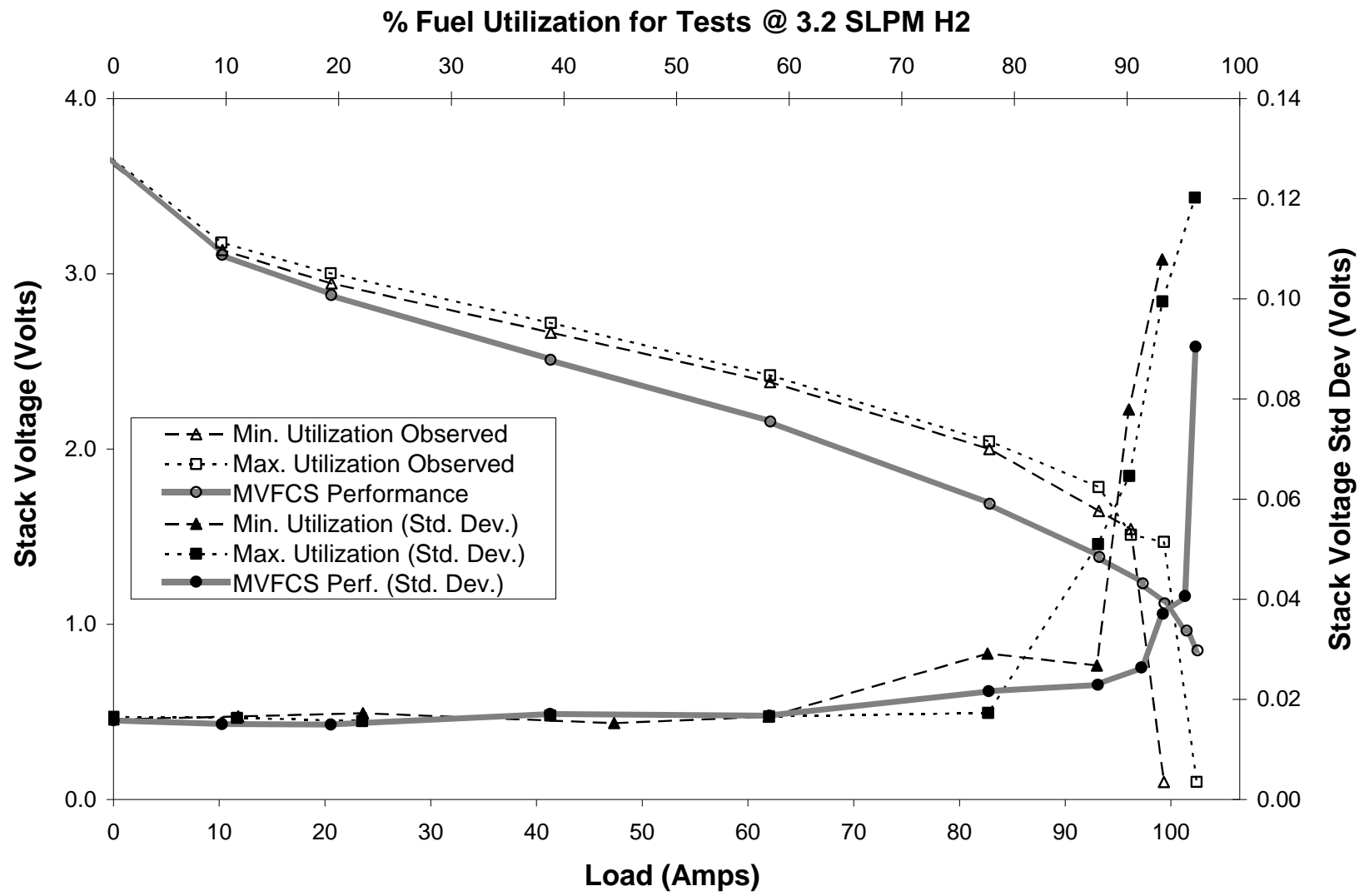

Fig. 4. Common manifold stack performance compared with MVFCS stack performance at a fuel flow rate of 3.2 SLPM - stack V-I curves and standard deviations of the stack voltages. 


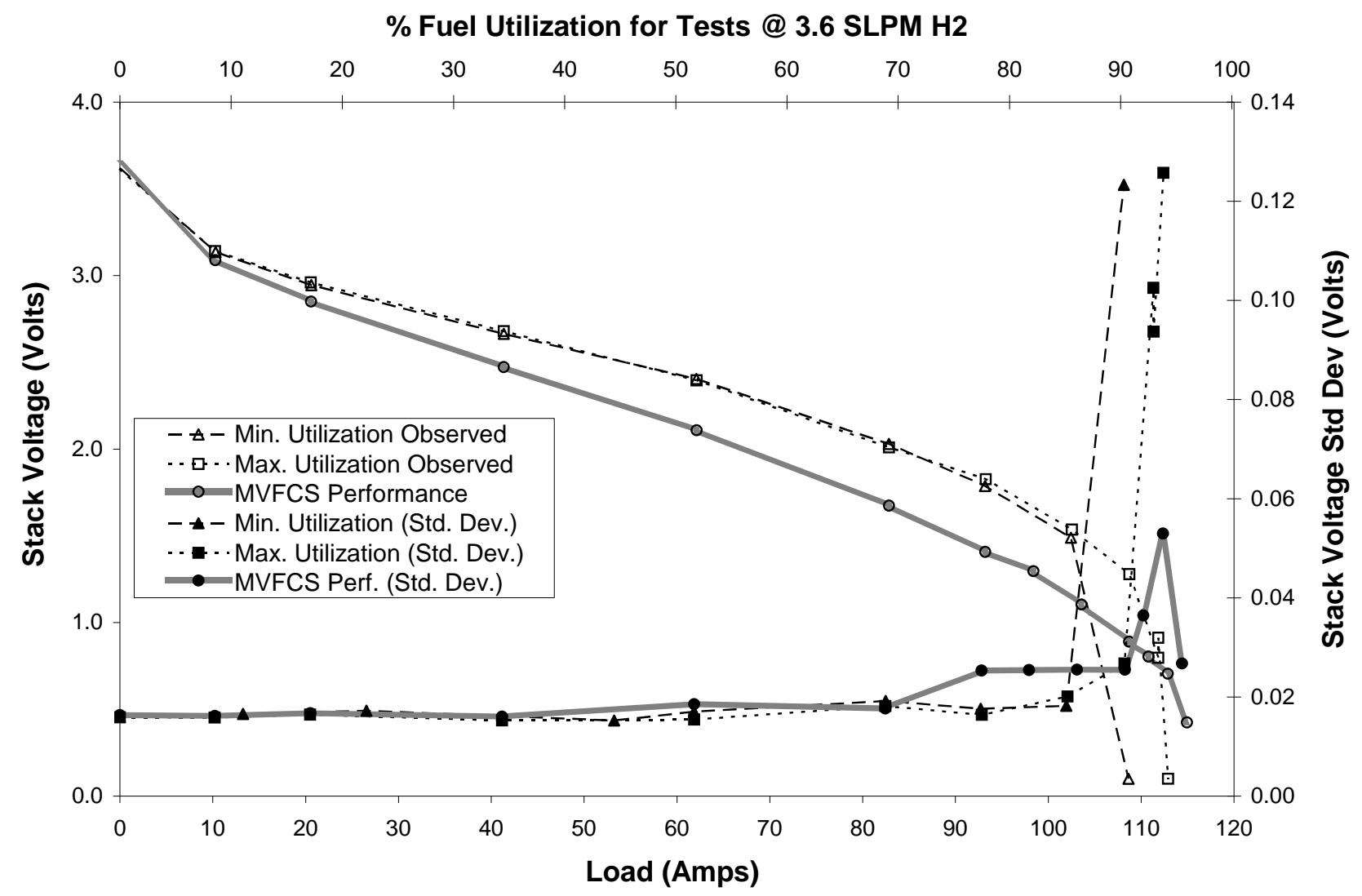

Fig. 5. Common manifold stack performance compared with MVFCS stack performance at a fuel flow rate of 3.6 SLPM - stack V-I curves and standard deviations of the stack voltages.

manifolds just as in the common manifold stack configuration (this can be seen in Figure 6). Since the focus of this study is on fuel flow control, only the 4 inlet fuel flows were manipulated in the experiments, while the air and cooling water valves were simply left wide open.

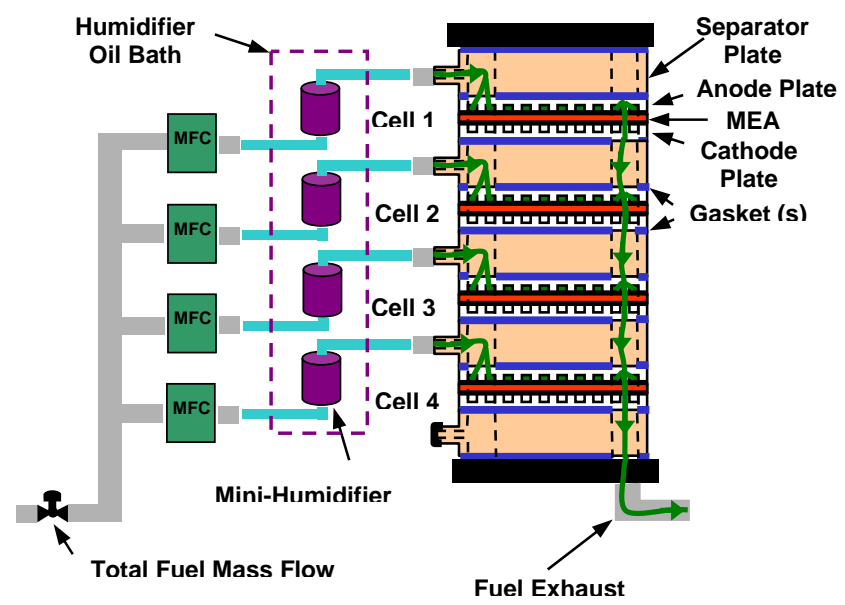

Fig. 6. Diagram of the mass flow control fuel delivery system.

\subsection{The fuel Mass Flow Control System (MFCS) experiments}

Unlike the common manifold stack setup, in the MFCS the hydrogen fuel is split into 4 separate control streams before the fuel is humidified. This needed to be done because mass flow controllers cannot reliably measure humidified gas flows. Thus, the 4 hydrogen streams had to be separately humidified before their introduction into the fuel cell. This was accomplished through the use of 4 "mini-humidifier" vessels that were corporately heated to 74 degrees $\mathrm{C}$ in a common oil bath to provide the necessary $100 \%$ relative humidity to the fuel flows. See Figure 6.

Flow mal-distribution tests were performed on the ICS with a total fuel flow of 3.6 SLPM and a total air flow of 16 SLPM. These rates were chosen to keep the maximum current density below 875 milliamps $\mathrm{cm}^{-2}$ and to provide adequate water management of the cathodes at this current density so that the membranes would not overheat and suffer damage. Tests were run under "balanced" flow conditions (0.9 SLPM of hydrogen to each cell) and under "unbalanced" conditions (0.8 SLPM of hydrogen to one cell and 0.93 SLPM of hydrogen to each of the other three cells-so that the total flow through the stack remained constant at 3.6 SLPM of $\mathrm{H}_{2}$ ). The 
purpose of these tests was to quantify the degree to which the V-I performance of the stack is affected by a single cell operating at fuel flow rates lower than those in the rest of the stack. Just as for the tests on the common manifold stack, time dependant statistical information on the stack voltage was calculated from the data.

Figure 7 summarizes the results of the fuel maldistribution tests conducted using the MFCS. Again, the standard deviation of the stack voltage is also shown to indicate how rapidly the stack voltage fluctuates at high fuel utilizations.
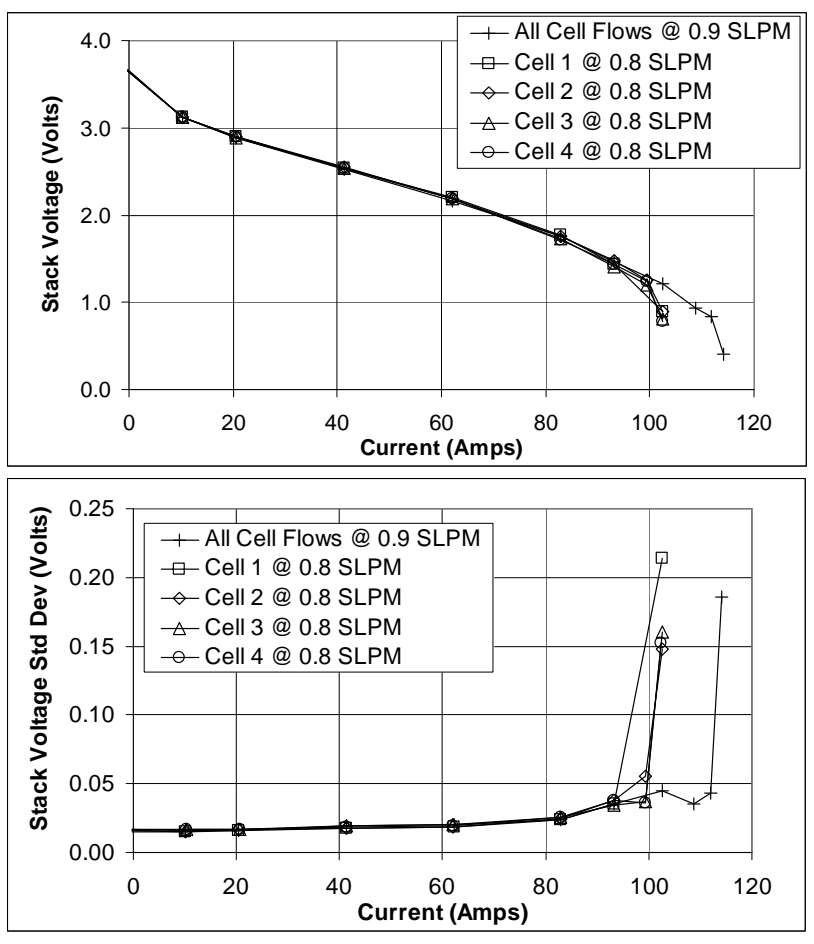

Fig. 7. MFCS-derived effects of fuel maldistribution on stack voltage @ 3.6 SLPM fuel flow.

\subsection{Micro-valve development}

Concurrent with the common manifold and MFCS work, NETL began an effort with the University of Pittsburgh to develop a novel MEMS-based micro-valve for cell-to-cell flow control in a PEMFC. The requirements for the fuel cell micro-valve include: (1) the ability to operate at less than 1psi in a hydrogen environment; (2) the ability to operate at elevated temperatures up to $120^{\circ} \mathrm{C}$ without any thermal actuation; (3) a small enough size to eventually be integrated within anode plate fuel channels; (4) fail at the nominal flow rate of 0.9 SLPM, rather than normally open or closed; (5) operate at nominal cell voltages. As shown below, not all of these requirements are yet achievable, and further development is necessary. Finally, simplicity, reliability, and robustness are other critical characteristics that will eventually need to be demonstrated.

The valve design is called the micro-piezoelectric orifice tap or $\mu$ PORT. A plate-like piezoelectric bender is used to actuate the valve by covering an orifice tap to varying degrees. The two-dimensional nature of the design evolved from an earlier micro-valve design that proved difficult to realize (Vipperman et al. [5]), and precludes the need for precise alignment of the orifice outlet and the actuator. The only critical dimension is the nominal stand-off distance between the top of the actuator and the bottom of the orifice surface, which controls the unactuated flow rate. A unique characteristic of this micro-valve is that the packaging is integrated into the valve design, rather than creating a valve inside of a package, which in turn has to be repackaged. The design is so forgiving of tolerances, commercial computer numerically controlled (CNC) machining could be used to create the integrated packaging. A similar approach could be taken when integrating these valves into the fuel cell plates.

Figure 8 below shows a picture of an assembled micro-valve prototype. The micro-valve consists of a 63 x 63 x 45mm machined polycarbonate block containing a lead zirconate titanate (PZT) bender actuator (PZT/aluminum/PZT sandwich). Although the prototype is large, most of the current design is packaging, and can be greatly decreased. Flow can move in either direction through the valve. The current design has one inlet/outlet on the face that contains the orifice, and the second inlet/outlet leaving the edge of the other side of the valve holder. The desired axial flow characteristic can be achieved by modifying the packaging.

From Figure 8, some of the internal components can be viewed through the body, including the O-ring seal and actuator along with its O-ring support. In contrast to the rather larger (63 x $63 \times 45 \mathrm{~mm}$ ) packaging, the size of the active portion of the valve is quite small $(30 \times 30 \times 0.4 \mathrm{~mm})$,

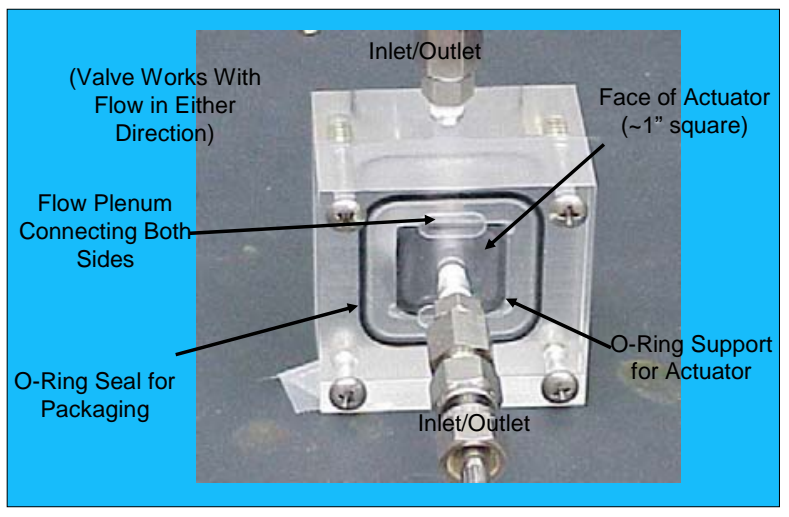

Fig. 8. Picture of assembled valve.

as shown by the profile view in Figure 9.

ANSYS Multiphysics software was used during the development to optimize the actuator parameters as well as to predict the flow performance and critical geometries for the valve. Ultimately, each valve was heuristically "tuned" by hand to provide the required nominal flow rate. 


\subsubsection{Micro-valve testing}

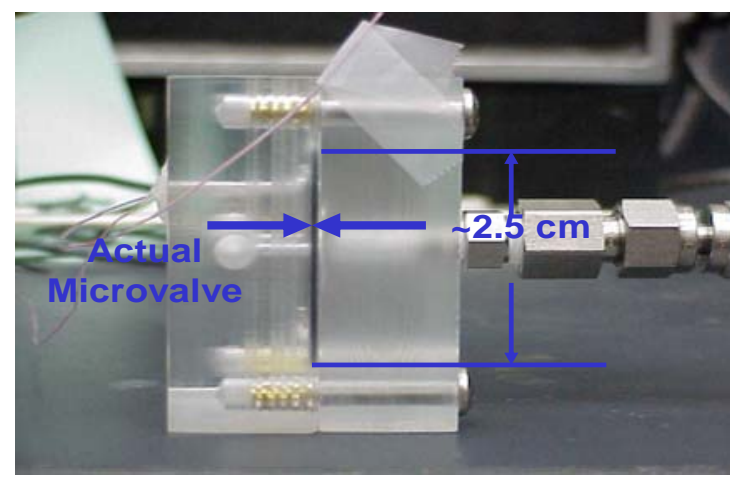

Fig. 9. Side view showing profile of valve actuator.

Two types of testing were conducted. The first was displacement testing of the actuators with an MTI-2100 photonic probe to ensure that they produced the predicted 20 microns of deflection for a 10 volt input. The second was flow testing. Thermal deflection testing of the actuators was performed in-situ by measuring any changes in flow rate versus changes in ambient temperature. A flow test loop was designed that consisted of an $\mathrm{H}_{2}$ supply, humidifier, flow meter, two pressure gauges, and a hot water bath to control the ambient temperature of the valve. Test results were obtained for three temperatures $\left(30,72\right.$, and $\left.100^{\circ} \mathrm{C}\right)$, three pressure drops across the valve $(3.4,5.2$, and $6.9 \mathrm{kPa})$, and 11 different voltages between -10 and 10 volts. Although the valve is capable of completely clamping off the flow with higher voltages and smaller actuator/orifice spacing, the valves were designed to modulate the flow by at least $\pm 20 \%$. The aggregate test results for one of the five valves across all three test temperatures are shown in Figure 10. From the small standard deviations indicated, the temperature effects on the flow rate are minimal an $d$ thermal actuation is very minimal.

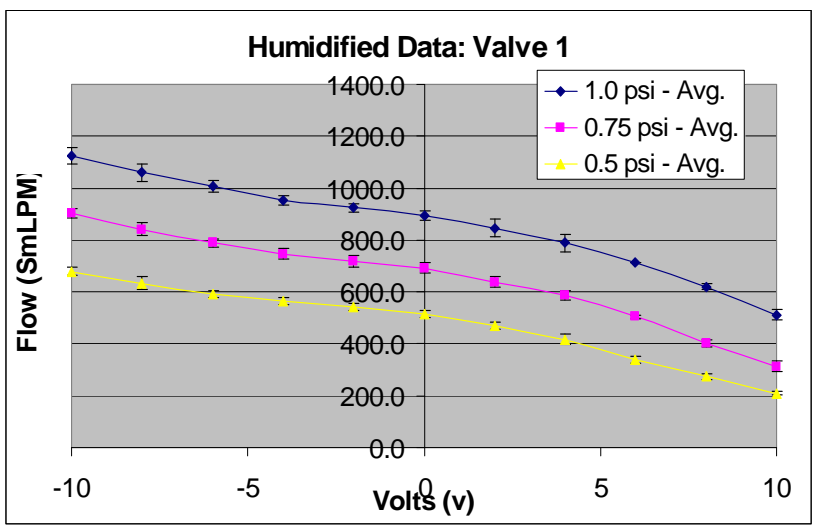

Figure 10. Flow data for a representative valve versus applied control voltage to actuator over all three temperatures.

\subsubsection{Micro-valve flow test summary}

Five valves have been constructed and tested. All five valves have been shown to meet the requirements for the application, namely consistent operation between 30 and $100^{\circ} \mathrm{C}$, operation in a hydrogen environment at $100 \%$ relative humidity, a nominal flow of 0.90 SLPM with a controllable range of at least 0.715-1.08 SLPM corresponding to a \pm 10 volt input. Although there was some variability between the flow characteristics of the five valves, they each were found to operate consistently at each pressure drop.

\subsection{Micro-valve flow control system experiments}

These micro-valves were used to implement a unique Micro-Valve Flow Control System or MVFCS. In this system the total fuel flow was again controlled by a single mass flow controller and passed through the same temperature-controlled fuel humidifier as was used in the common manifold stack experiments. From the humidifier the flow was carried through heat-traced lines to a heated external fuel manifold from which it split into the four micro-valve controlled and heat-traced streams that provided fuel to the individual cells via connections on the separator plates. See Figures 11 and 12. Again, the outlets from each cell empty into internal stack manifolds just as in the common manifold stack configuration (this can be seen in Figure 12).

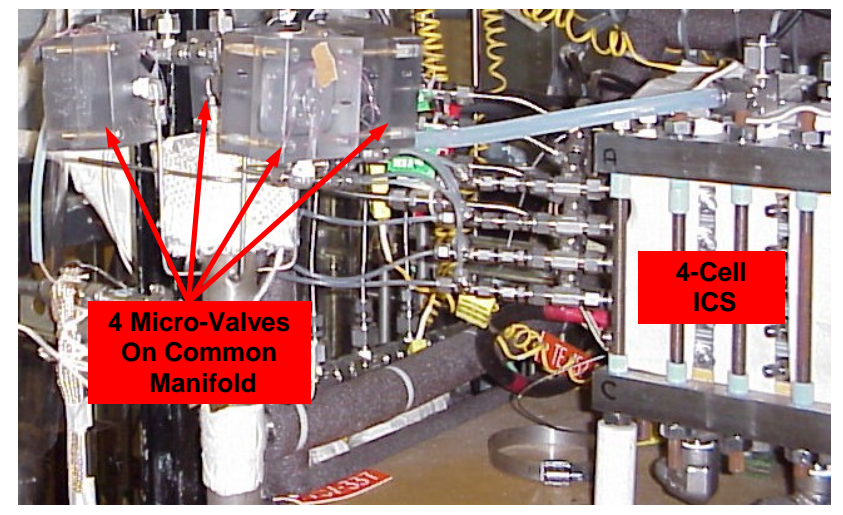

Fig. 11. Photo of the ICS/micro-valve fuel manifold system.

To demonstrate the potential benefits of the FCEM technique for operating a fuel cell at the highest possible fuel utilizations, proper installation of the micro-valves was critical. The torque on each of the 4 housing bolts (that hold the two halves of the valve body together) needed to be carefully adjusted to provide as balanced a flow split between the 4 cells of the stack as possible with the valves in their unactuated (nominal) state. Adjustment success was measured by the maximum fuel utilization that could be achieved running FCEM experiment V-I curves without actuating the microvalves. Values from $92 \%$ to $94 \%$ fuel utilization were achieved. It was assumed that any flow imbalance caused by the presence of the micro-valves, given this degree of 
initial flow balance, could be easily overcome by the micro-valves in their operating mode.

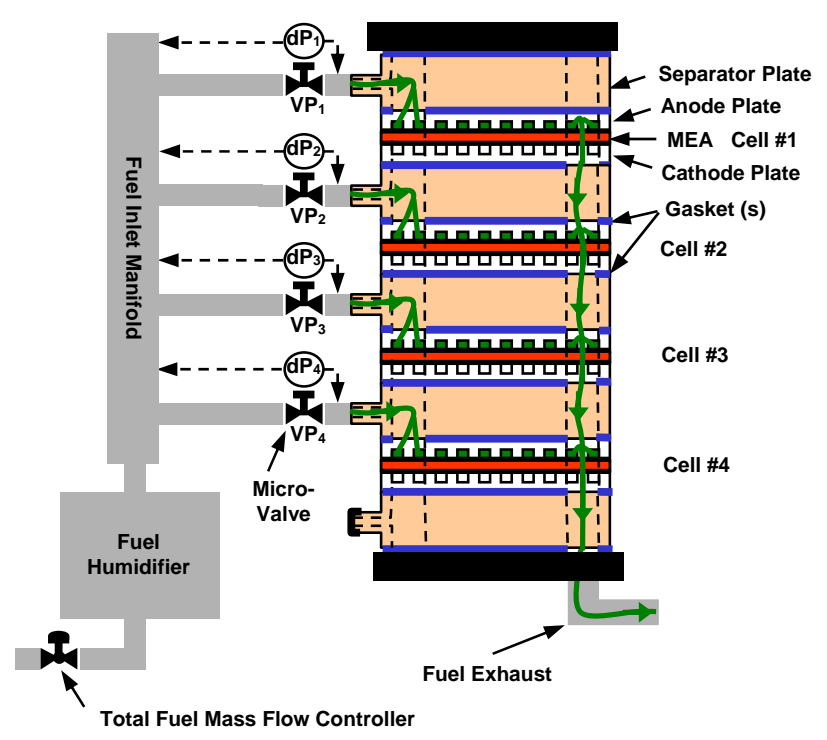

Fig. 12. Diagram of the micro-valve fuel control system with ICS.

The control strategy employed was a very simple one: Open-loop, flow-on-demand. That is, the microvalve control voltage was based on the cell voltage. If the cell voltage dropped then the valve opened proportionately to allow more fuel flow to the cell in an attempt to deal with the low voltage condition. The valves were each set up to go totally open when the cell voltage dropped to 0.25 volts and to go as closed as possible when the cell voltage was 1.0 volts. Note that the micro-valves, even at "fully closed" wouldn't actually shut off the flow to a cell, they would just increase the flow resistance to that cell. This strategy was chosen for a number of reasons. First, it is very simple, which minimizes system cost and complexity. Second, it is not necessary to actually measure the flow through the valve, which, given the humidified nature of the fuel, would not be easily achieved. Finally, this technique might eventually be refined enough to allow the micro-valve to actually derive both its power and its control signal from the cell voltage - making embedded micro-valves inside fuel cells much more feasible. Stack and individual cell V-I curves were collected using this configuration at the same three stack fuel flow rates that were used in the common manifold stack tests - 2.8, 3.2, and 3.6 SLPM of $\mathrm{H}_{2}$. The stack voltages and standard deviations of the stack voltages for these three cases as well as the corresponding minimum and maximum fuel utilization data of the common manifold stack tests were presented earlier in Figures 3-5. Note that on each of these three graphs, $100 \%$ fuel utilization (at the given fuel flow rate) occurs at the far right side of the graph - that is, the highest load value show on the $\mathrm{x}$-axis is the value at which $100 \%$ fuel utilization would theoretically occur.

\section{Discussion of experimental results}

\subsection{Fuel mal-distribution results}

The data shown in Figure 7 demonstrates the degree to which fuel mal-distribution between cells can affect overall stack performance. In these tests the flow to a single cell was decreased by $11 \%$ (from 0.9 to 0.8 SLPM). The maximum achievable load current dropped from 114 to 102 amps - a reduction of $10 \%$. This result was consistent among each of the 4 cells and shows the significant effect that fuel mal-distribution can have on stack performance. Thus it is apparent that the need to keep fuel flows balanced between the cells is critical to achieving the best possible stack fuel utilization.

\subsection{Comparison of MVFCS and common manifold stack results}

The data from the common manifold stack shown in Figures 3-5 provides performance results typical of PEMFCs. The standard deviation of the stack voltage data indicates the extent to which this voltage fluctuates as load increases, and these fluctuations, at the individual cell level, are what the FCEM technique was developed to minimize in order to extend the fuel cell's performance to higher fuel utilizations. Also, this data provides an example of how variable the fuel utilization of a common manifold stack can be: For instance, at a flow rate of 2.8 SLPM $\mathrm{H}_{2}$ the common manifold stack's fuel utilization varied between 90 and 100 percent. At 3.2 SLPM the utilization varied between 90 and 96 percent, and at 3.6 SLPM it varied between 85 and 93 percent. Based on the fuel mal-distribution results reported above, it appears that a 10 percent fuel mal-distribution is not uncommon in a common manifold stack. This data provides a base-line of comparison between common manifold stack performance and the FCEM performance achieved while using the MVFCS.

Figures 3-5 also show the maximum fuel utilization benefits that the MVFCS was able to provide from a stack voltage perspective. That is, the MVFCS stack performance curves are shown in comparison with the minimum and maximum fuel utilization performances of the common manifold stack at the three flow rates used in this study. At a flow rate of $2.8 \mathrm{SLPM} \mathrm{H}_{2}$ the common manifold stack's minimum observed fuel utilization occurred at some point between 90 and 93 percent. The MVFCS, on the other hand, produced a fuel utilization of 96 percent for an improvement of 3 to 6 percent. At 3.2 SLPM $\mathrm{H}_{2}$ the common manifold stack's minimum observed fuel utilization occurred once again between 90 and 93 percent. The MVFCS again produced 96 percent utilization for another improvement of 3 to 6 percent. At 3.6 SLPM $\mathrm{H}_{2}$ the common manifold stack's minimum observed fuel utilization occurred between 86 and 91 


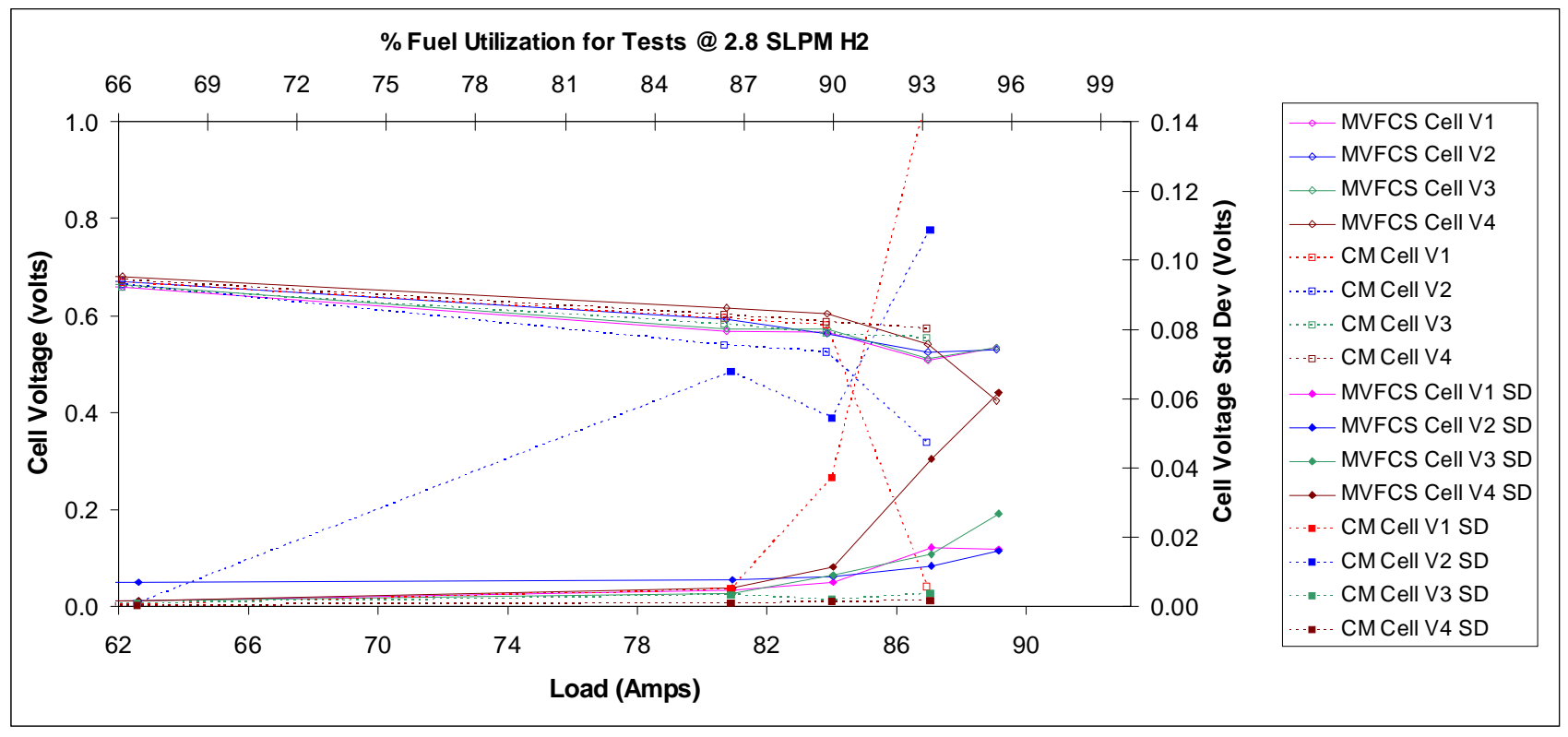

Fig. 13. Cell voltage-based $\mathrm{H}_{2}$ utilization improvement evaluation @ 2.8 SLPM.

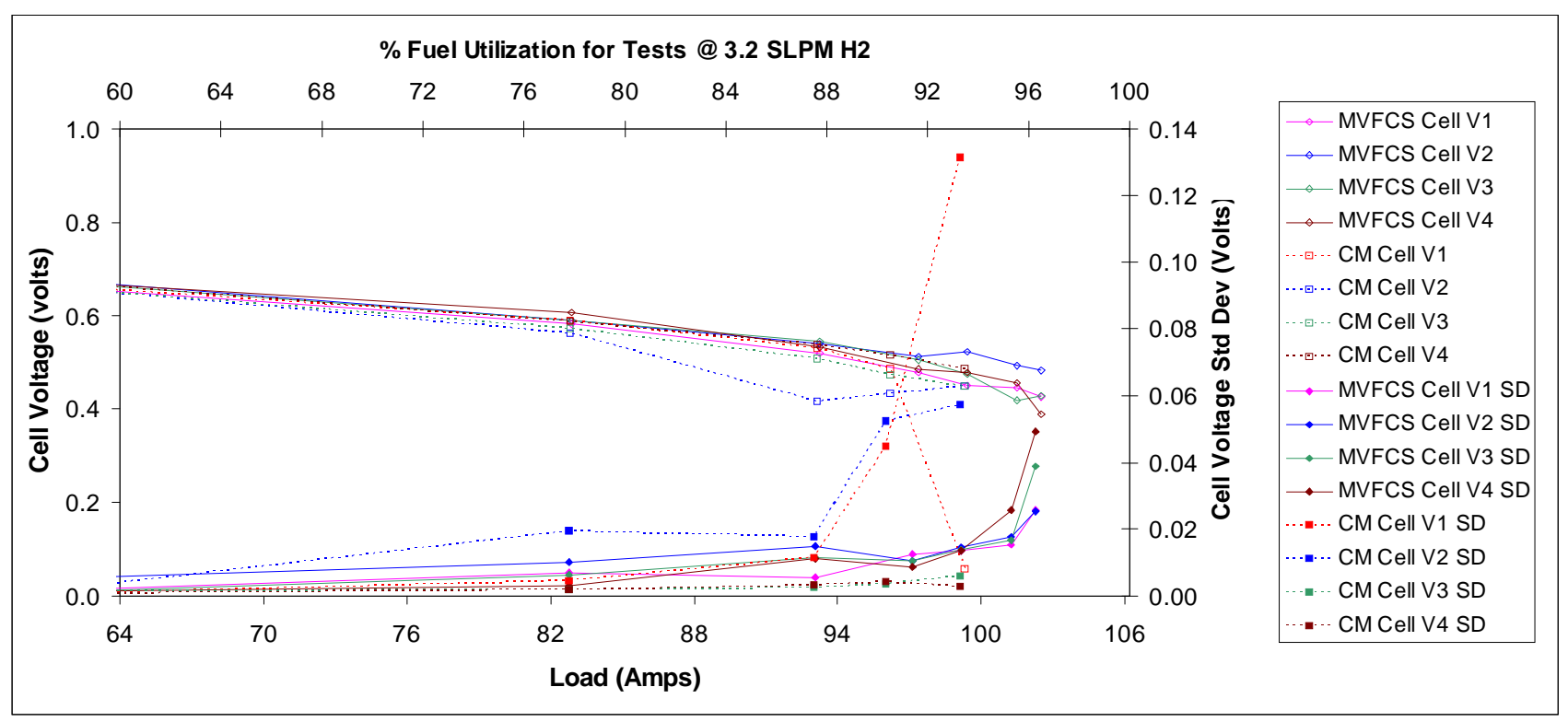

Fig. 14. Cell voltage-based $\mathrm{H}_{2}$ utilization improvement evaluation @ 3.2 SLPM.

percent, as compared to another MVFCS performance of 96 percent, yielding an improvement of 5 to 10 percent. Note that the difference in slope between the MVFCS and the common manifold data is a by-product of the greater ohmic losses in the ICS due to the thick separator plates that were used (refer to Figure 12). Assuming that the observed common manifold data are "typical", in the sense that the minimum observed fuel utilizations tend to occur with regularity, this method of comparing the
MVFCS and common manifold stack data yield a minimum overall fuel utilization performance improvement of 3 percent and a potential maximum of 10 percent. However, this large spread in potential improvements is in part a result of the size of the load steps taken during data collection.

Another, and perhaps better, method of assessing the performance benefits of the FCEM technique can be derived from the data shown in Figures 13-15. In these 


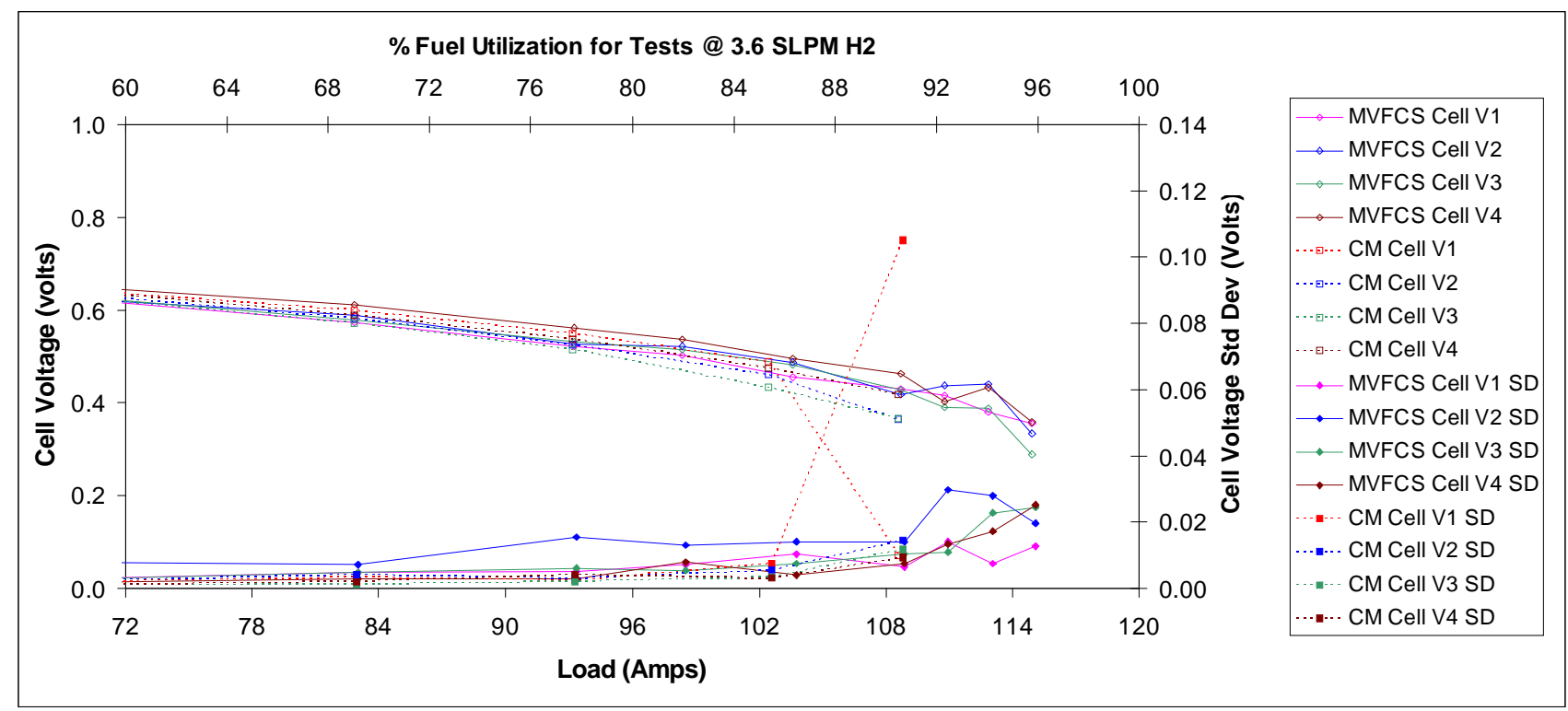

Fig. 15. Cell voltage-based $\mathrm{H}_{2}$ utilization improvement evaluation @ 3.6 SLPM.

figures individual cell voltage data from both the MVFCS tests and from the common manifold stack tests, at the same operating conditions, are simultaneously displayed. This data includes: 1 ) the cell voltages from all 4 cells of the ICS, gathered while testing the MVFCS at each of the three tested fuel flow rates; 2) the cell voltages for the common manifold tests that exhibited the minimum observed fuel utilizations at each of the three tested fuel flow rates, and 3) the standard deviations of all of the above-mentioned cell voltages. A secondary $\mathrm{x}$-axis (as was used in Figures 3-5) provides a scale showing the percent fuel utilizations for each of the load currents shown on the primary x-axis. To quantify the performance benefit of the MVFCS over conventional common manifold stacks, consider the common manifold cell voltage standard deviation curves on each of these graphs. As these curves indicate, the amount of cell voltage fluctuation that occurs as the stack is pushed to its maximum load capability begins to increase exponentially. From the gathered data, it can be noted that if a cell voltage standard deviation exceeded 0.08 volts, then that cell would "crash" in the sense that its voltage would eventually drop below 0.1 volts, forcing an end to the test in order to protect the cell's integrity. Let the maximum fuel utilization value for each of the common manifold stack tests be arbitrarily defined as the fuel utilization value that was measured as this 0.08 cell voltage standard deviation value was reached. Note that the MVFCS cell voltages show noticeably increased fluctuations from this fuel utilization point on until the maximum load/utilization is reached. It is this level of fluctuation, one significant enough to "crash" a cell in the common manifold stack, that the MVFCS must be able to mitigate in order to extend the fuel utilization capability of the stack. Using the definitions provided above, the data in these three figures can be used to determine the amount of fuel utilization performance improvement that the MVFCS provided over the common manifold stack. For a stack flow rate of $2.8 \mathrm{SLPM} \mathrm{H}_{2}$ (or 0.7 SLPM per cell) the common manifold stack performed reliably up to 92\% fuel utilization while the MVFCS achieved 96\%. For a stack flow rate of $3.2 \mathrm{SLPM} \mathrm{H}_{2}$ (or 0.8 SLPM per cell) the common manifold stack again performed reliably up to $92 \%$ fuel utilization while the MVFCS achieved $96 \%$, and for a stack flow rate of $3.6 \mathrm{SLPM} \mathrm{H}_{2}$ (or 0.9 SLPM per cell) the common manifold stack performed reliably up to $91 \%$ fuel utilization while the MVFCS again achieved $96 \%$. Thus the overall improvement was between 4 and 5 percent, and since the minimum observed common manifold fuel utilizations were used in this analysis, this is the maximum improvement that can be expected, based on this data.

In all, the MVFCS consistently achieved 96 percent fuel utilization at all of the tested fuel flow rates. Given the highly variable nature of the common manifold stack performance, this is a significant performance improvement. The usefulness of cell-to-cell flow balancing to operate PEMFCs at the highest possible fuel utilization is apparent, and MEMS micro-valves can provide the technological means to achieve this flow balancing.

\section{Conclusions}

By comparing the performance of balanced fuel flow PEMFC stacks to unbalanced, this work has demonstrated some of the possible benefits that can be obtained in PEMFCs by using cell-to-cell flow distribution control. The data have shown that a significant drop in fuel flow to even a single cell will cause a significant drop in the 
maximum load that the stack can support. By providing individual cell flow control, (or ultimately individual micro-channel, within the cell, flow control) cell performance can be optimized so that the additional flow required for reliable stack operation can be reduced below typically-used levels, thereby improving fuel efficiency. In addition, by using micro-systems manufacturing techniques, the additional capital cost for the micro-valves to perform the flow balancing could be low; and eventually, it may be possible to operate such microvalves directly off of the cell voltage, minimizing parasitic power consumption and peripheral hardware.

\section{Future Work}

Ultimately, internal dynamic balancing of individual cell fuel flows is desired to achieve reliable stack performance at high fuel utilization. Work is presently being performed to investigate the development of higher temperature micro-valves that could provide this internal dynamic balancing for SOFCs.

\section{References}

[1] McGarry, M., Grega, L., 2006, Effects of Inlet Mass Flow Distribution and Magnitude on Reactant Distribution for PEM Fuel Cells, J. of Fuel Science and Technology, Vol. 3, pp. 45-50.

[2] Lee, H.I., C.H. Lee, T.Y. Oh, S.G. Choi, I.W. Park, K.K. Baek, 2002, Development of $1 \mathrm{~kW}$ class polymer electrolyte membrane fuel cell power generation system, J. of Power Sources, Vol. 107, pp. 110-119.

[3] Voss, H. H., D. P. Wilkinson, P. G. Pickup, M C. Johnson, V. Basura, 1995, Anode Water Removal: A Water Management and Diagnostic Technique for Solid Polymer Fuel Cells, Electrochimica Acta, Vol. 40, No. 3, pp. 321-328.

[4] Mallant, R. K. A. M., 2003, PEMFC systems: the need for high temperature polymers as a consequence of PEMFC water and heat management, J. of Power Sources, Vol. 118, pp. 424-429.

[5] Vipperman, J.S., Ayhan, A.F., Clark, W.W., Thornton, J., Gemmen, R.S., 2003, Fabrication and Preliminary Testing of a Novel Piezoelectrically Actuated Microvalve, Proceedings of IMECE-03, Washington, D.C.. 